\title{
Improvised Guard for Next Level Security Automated Vehicle Security system using Artificial intelligence and Convolutional Neural Network
}

\author{
Joel M John ${ }^{1}$, Noel Phillip Issac ${ }^{1}$, \\ Jerin Thomas ${ }^{1}$, Subin Alexander ${ }^{1}$ \\ Department of Computer Science and Engineering, \\ St Thomas College of Engineering and Technology \\ Chengannur, India
}

\author{
Syamraj B S ${ }^{2}$ \\ ${ }^{2}$ Assistant Professor \\ Department of Computer Science and Engineering, \\ St Thomas College of Engineering and Technology \\ Chengannur, India
}

\begin{abstract}
This paper details fully automated vehicle security system involving vehicle model, make detection, driver face recognition and parking system guided by a virtual assistant. The core technology of the system is built using a sequence of deep Convolutional Neural Networks (CNNs). This system performs face recognition of the driver and vehicle model, make detection and permit access by opening barrier gate. This allows bigger organizations to control and monitor vehicle traffic as well as gain user data for security purpose. For quantitive analysis, we show that our system outperforms the leading vehicle security system. Proposed paper project website is also available at $\mathrm{http}: / / \mathrm{www}$.astound.ga/igns
\end{abstract}

Keywords-Virtual Assistant, Recognition, Authorization, Validation, Vehicle, User interaction

\section{INTRODUCTION}

Recently CCTV security systems is used in numerous areas, industries, entry restricted areas or by government including ANPR(Automatic Number Plate Recognition) for vehicles, Face identification etc.. ANPR Security Systems (Automatic Number Plate Recognition) can be used to store the images captured, as well as the text from the number plate. It is also able to store a photograph of the driver. With the advent and enhancements of deep CNNs and cheaper, faster processing hardware, we need to take a fresh look at the problem. Advances in the design of CNNs have resulted in significant increases in performance accuracies for many tasks. In this work, we harness the power of CNNs in an end-to-end system capable of detecting and recognizing license plates with low error rates. We present a system that is robust to variations in conditions (camera movement, camera angle, lighting, occlusion, etc) and license plate templates (size, designs, formats, etc). But the existing system needs much more relations, tight security based information technologies and additional automated features for easy access and user interactions .That's where this project comes in handy, IGNS (Improvised Guard for Next Level Security) system provides entirely automated vehicle security by a virtual assistant involving user interactions by clearly recognizing the driver with face recognition and vehicle model, color, make, number plate recognition differentiating a genuine or fake number plate by verification with MVD(Motor Vehicle Department) database. This system enhances apt Vehicle security and requires no manual control as it is fully automated functional system monitored and controlled by the Artificial Intelligent system.

\section{LITERATURE SURVEY}

1. Number Plate Recognition for Use in Different Countries Using and Improved Segmentation

Automatic Number Plate Recognition (ANPR) is a real time embedded system which identifies the characters directly from the image of the license plate. It is an active area of research. ANPR systems are very useful to the law enforcement agencies as the need for Radio Frequency Identification tags and similar equipments are minimized. Since number plate guidelines are not strictly practiced everywhere, it often becomes difficult to correctly identify the non-standard number plate characters. In this paper we try to address this problem of ANPR by using a pixel based segmentation algorithm of the alphanumeric characters in the license plate. The non-adherence of the system to any particular country-specific standard \& fonts effectively means that this system can be used in many different countries - a feature which can be especially useful for trans- border traffic e.g. use in country borders etc.

\section{A Study of Car Park Control System Using Optical Character Recognition}

This paper presents the study and design of car park control system using optical character recognition (OCR) devices. The system uses client server environment. The administrator will monitor the system and the database from the server side. Furthermore the parking information will be displayed static based on the database shared by the server. Server application and database will be stored in the server. The result shows the system is capable to save log record that will ease tracking parking user, updating user and parking credit database as well as monitoring availability of parking spaces.

\section{Prevent Crime by Face Recognition}

Face recognition is currently being used to instantly identify when known shoplifters, organized retail criminals or people with a history of fraud enter retail establishments. Photographs of individuals can be matched against large databases of criminals so that loss prevention and retail security professionals can be instantly notified when a shopper enters a store that prevents a threat. Face 
recognition systems are already radically reducing retail crime.

\section{PROPOSED SYSTEM}

IGNS (Improvised Guard for Next Level Security) system provides apt security by a virtual assistant involving user interactions by clearly recognizing the driver with face-id and vehicle model, color, make, number plate differentiating a genuine or fake number plate by comparison with MVD(Motor Vehicle Department) database. The contributions of this system are summarized below.

- Face recognition involving face identification of the driver

- Vehicle model, make, color detection

- User authentication, involving validation of number plate and driver details such as name, mobile no.

- System action involving access or denial

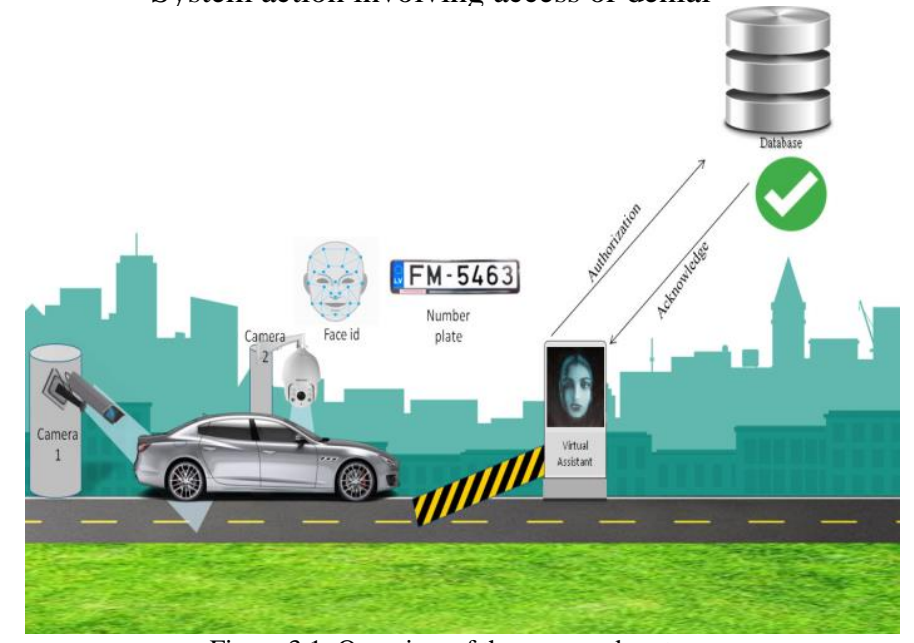

Figure 3.1: Overview of the proposed system

IGNS security system strives for automation hence the entire system control is based on the guidance of an Artificial intelligence or Virtual Assistant named A. I. D. A (Advanced Intelligence in Decision and Action) which enhances interaction with the driver.

\section{Virtual Assistant}

- Once Recognition is initiated by user, then AIDA undertakes the system control and communicates with the user and extracts information of the driver through voice recognition such as driver name and mobile number.

- Meanwhile the camera1 and camera 2 capture images of the face of driver and vehicle respectively

- AIDA is powered by speech recognition system which precisely with an accuracy of $95 \%$ can recognize and understand speech.

- This provides way for interaction of the system with the driver for accessing information such as name, mobile number for future security purposes.

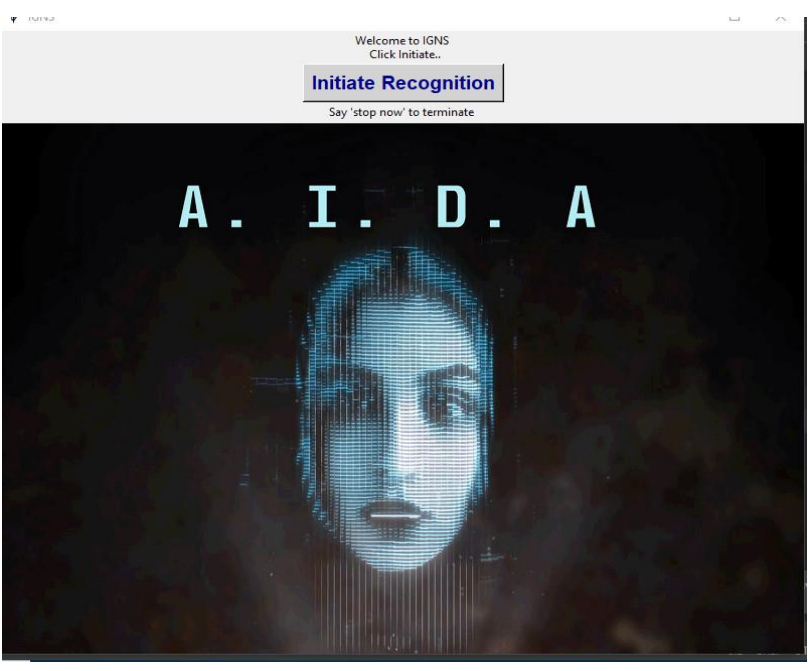

Figure 1.2 user interface of virtual assistant

\section{Recognition}

\subsection{Face Recognition}

Face recognition systems use computer algorithms to pick out specific, distinctive details about a person's face. These details, such as distance between the eyes or shape of the chin, are then converted into a mathematical representation and compared to data on other faces collected in a face recognition database. Based on the information received from the face recognition the driver can be identified as an existing user or a unknown(new user).

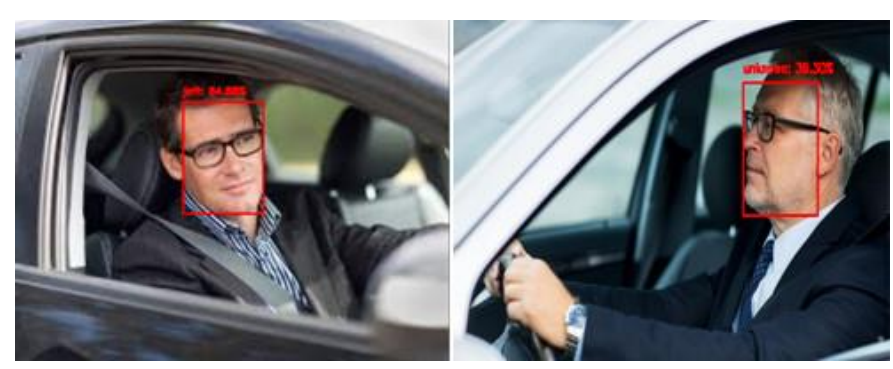

Figure 2.1.1 Recognized Driver

\subsection{Vehicle Recognition}

Make, model and color recognition (MMCR) of vehicles is one of great interest in several applications such as lawenforcement, driver assistance, surveillance and traffic monitoring. The IGNS system detects vehicle plate, model and make in order to validate the authenticity of the vehicle with the number plate from the MVD.

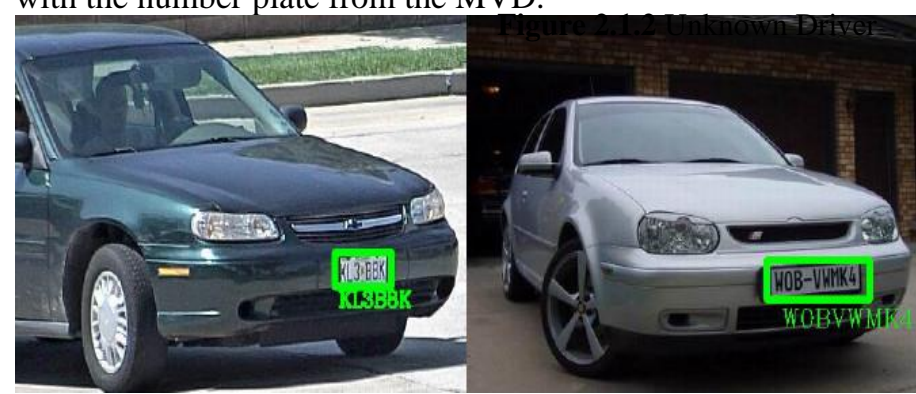

Figure 2.2.1 Vehicle Recognition 


\section{Authorization}

Authorization is a function of providing access or privilege to the user based on the following factors. 1)Existing user 2)Genuine Number plate

\section{1)Existing user}

Based on the information received from the face recognition the driver can be identified as an existing user or a unknown(new user) as shown in Fig 2.1.1 and Fig 2.1.2. If the driver face is existing user then the driver and the vehicle will be granted access only if the number plate is genuine. On the other hand if the driver face is recognized as unknown then the system will prompt for user registration followed by saving the driver name, mobile number and face id for future recognition and proceed to number plate verification.

\section{2) Genuine Number plate}

The system scans the number plate and initiates verification with the MVD database. Henceforth changes found different in vehicle color, make from the actual MVD database can be identified easily and the number plate will be blacklisted and access will be denied.

\section{3) Parking Management}

If the system verified number plate is genuine then the access is granted to the vehicle and an welcome SMS is sent assigning a parking slot for parking. Parking system assigns parking slot with respect to the vehicle model.

\section{SYSTEM ARCHITECTURE}

The given is the conceptual architectural diagram of the IGNS system.

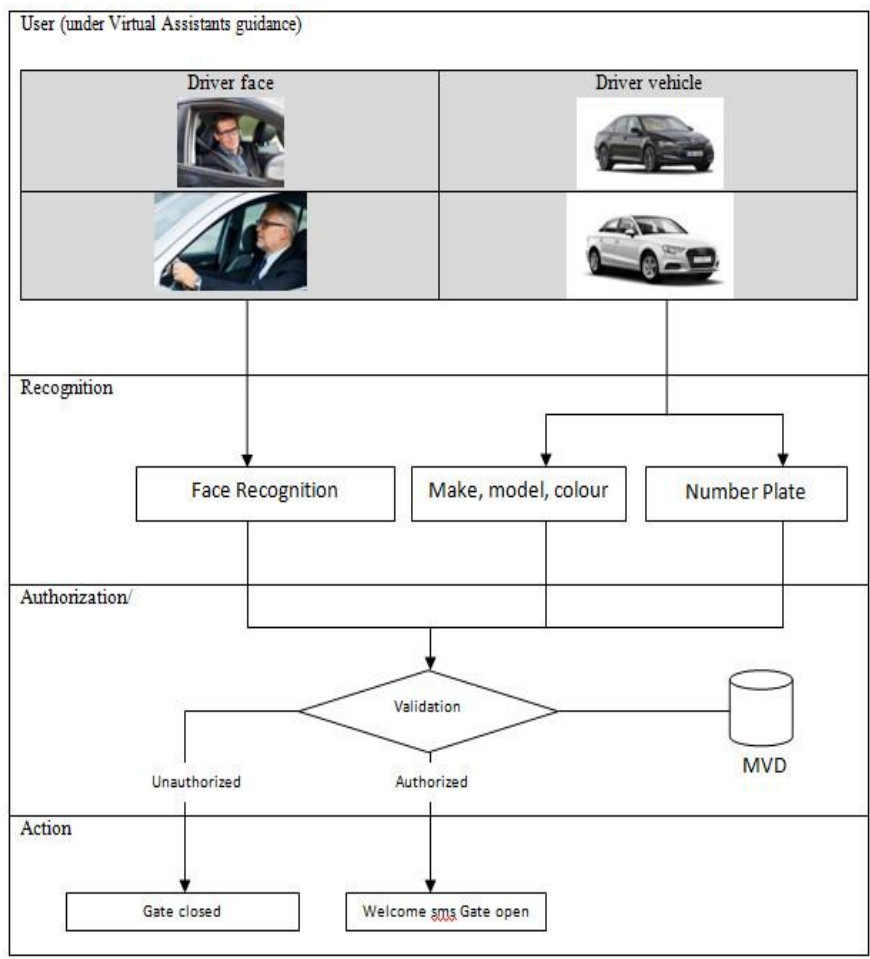

Figure 4.1 System Architecture of IGNS security system
- Phase 1: Once the system is initiated to start recognition the virtual assistant AIDA takes control by taking pictures of the vehicle as well the driver face. This was the first user interaction phase.

- $\quad$ Phase 2:The next is the recognition phase where the face and vehicle picture collected from the system is detected, transformed and cropped and feed to deep neural network. Here the system performs recognition of driver and vehicle recognition such as number plate, make, and color.

- Phase 3:The third phase is authorization where the information received from the recognition are strictly validated with the system database containing information of all users as well as MVD database. Here the system can identify user and detect fake number plates. Based on the results the system will conclude as authorized or unauthorized vehicle and proceed to final Action.

- $\quad$ Phase 5:The Final is Action phase where the user will receive an welcome sms and the gate will be opened for the driver to proceed if its an authorized user or else otherwise.

\subsection{System Requirements}

\subsubsection{Minimum Hardware Requirements}

- CPU: Core i5

- GPU: Geforce GTX 750 Ti

- RAM: 8GB

- Hard Disk: 128GB

- Arduino Uno

\subsubsection{Software Requirements}

- OS: Windows

- Coding Language: Python 3 or higher, $\mathrm{C}$

- IDE: Pycharm 2019 or higher, Arduino 1.0

\section{RESULTS AND DISCUSSION}

For a fully investigation of the proposed methods, the proposed system considers the following aspects:

- Effectiveness: The studies evaluate the detection performance of the trained model and comparison methods in terms of Accuracy, Recall and Precision

- Efficiency: The studies evaluate efficiency of the methods by comparing the CPU time of training each model. All experiments are performed on an x64 machine with $2.80 \mathrm{GHz}$ Intel Core i5 CPU and $16 \mathrm{~GB}$ RAM.

The IGNS vehicle security system performs authorization of user involving:

- $\quad$ Speech Recognition: A.I.D.A Recognizing speech from driver and exchange information.

- Face id: Recognizing driver face for identification 
- Vehicle Recognition: Recognizing driver vehicle details

The IGNS system allows admin to control and monitor the vehicles. Figure 5.1 represents admin view of IGNS system

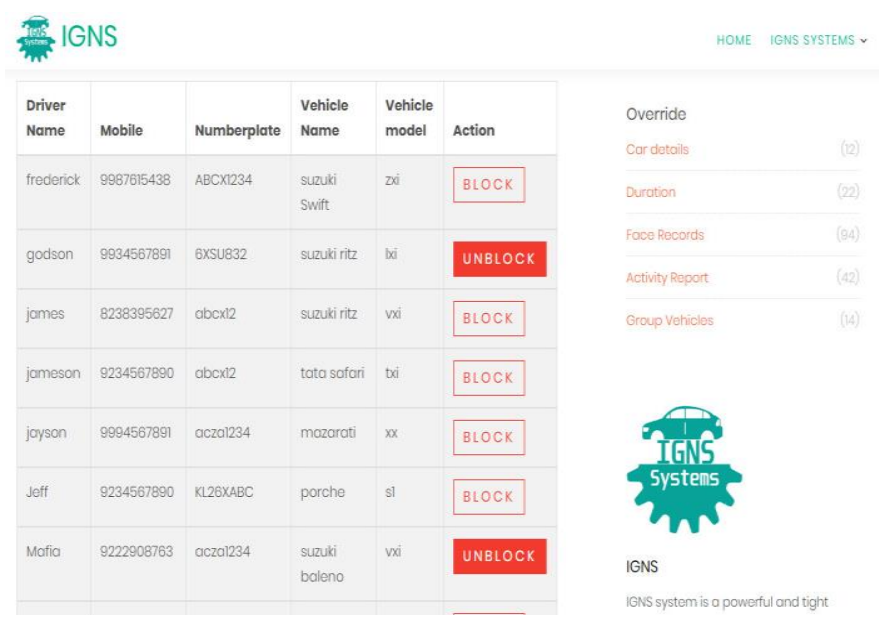

Figure 5.1 Admin View of IGNS system

The IGNS system has read access over the MVD hence it helps the system to identify fake number plate of the vehicle. The figure 5.2 represents the view of the MVD database.

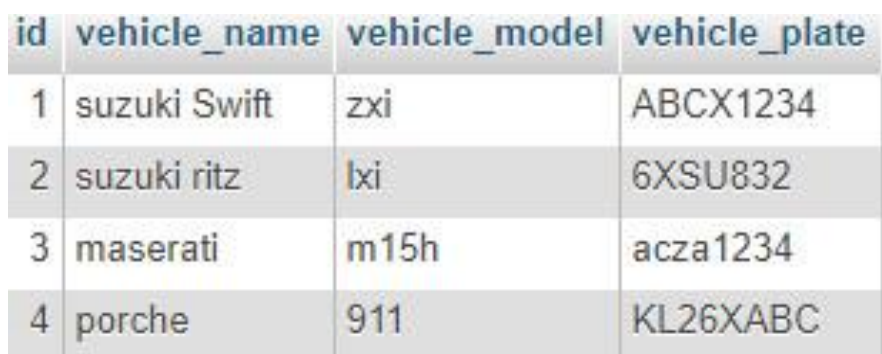

Figure 5.2 Motor Vehicle Department Database

\section{CONCLUSION AND FUTURE SCOPE}

In this paper we presented an end to end autonomous advanced vehicle security system for vehicles recognizing make, model, color recognition as well as driver face recognition. The approach to the design and implementation of deep neural networks and a sizable dataset for training allow to label vehicles in real time with high degrees of accuracy. With the additional features of face recognition of drivers, validation of number plates powered by A.I.D.A the IGNS system proposes fully automated functional security system that initiates a tight security.

\subsection{Advantages and Limitations}

Advantages

- Automated Vitual Assistant system for interaction with user.

- No need of an actual security guard
- Fully autonomous security

- Reduces fraudulent activities of number plates

- Automated system for detecting vehicles models

- $\quad$ Automated Parking slots via sms

Limitations

- New user registration may consume some time

- Number plate recognition may consume more time on difficult fonts

- Weather condition may affect the clarity of pictures captured by camera

- Heavy Noise during speech recognition may result error values

\subsection{Future Applications}

1. Road Safety: Features of IGNS system such as automated number plate recognition system combined with face recognition and vehicle detection can be upgraded to new level of security for road safety management system for the government. The system can be used to understand and analyze daily traffic flow.

2. Vehicle Tracking: Since the Proposed system uses number plate for recognition it is possible to track the vehicle anywhere with the same plate.

3. High Security: The system can work efficiently in areas which restrict human interactions such as military base.

4. Monitor Activity: The system can be used to monitor activity records of the driver and vehicle which would stand as a vital information for investigation authorities.

5. User Tracking: The system enables face identification for security purpose. Hence can play a vital role in tracking a user.

\section{REFERENCES}

[1]. Espresso: A Fast End-to-End Neural Speech Recognition Toolkit Yiming Wang; Tongfei Chen ; Hainan Xu ; Shuoyang Ding ; Hang Lv ; Yiwen Shao ; Nanyun Peng ; Lei Xie ; Shinji Watanabe ; Sanjeev Khudanpur 2019 IEEE Automatic Speech Recognition and Understanding Workshop (ASRU)

[2]. Sochor, J., Herout, A., Havel., J.: Boxcars: 3d boxes as cnn input for improved_ne-grained vehicle recognition. In: CVPR. (2016)

[3]. Hsieh, J.W., Chen, L.C., Chen, D.Y.: Symmetrical surf and its applications to vehicle detection and vehicle make and model recognition. In: IEEE Transactions on intelligent transportation systems. (2014)

[4]. Gu, H.Z., Lee., S.Y.: Car model recognition by utilizing symmetric property to overcome severe pose variation. In: Machine vision and applications. (2013)

[5]. Yang, Linjie, e.a.: A large-scale car dataset for _ne-grained categorization and veri_cation. In: Proceedings of the IEEE Conference on Computer Vision and Pattern Recognition. (2015)

[6]. Lin, T.Y., RoyChowdhury, A., Maji., S.: Bilinear cnn models for _ne-grained visual recognition. In: Proceedings of the IEEE International Conference on Computer Vision. (2015) 
[7]. Du, S., Ibrahim, M., Shehata, M., Member, S., Badawy, W.: Automatic license plate recognition (alpr): A state-of-the-art review. In: IEEE Transactions on Cir- cuits and Systems for Video Technology. Volume 23. (2013)

[8]. Cheang, T.K., Chong, Y.S., Tay, Y.H.: Segmentation-free vehicle license plate recognition using convnet-rnn. (2017)

[9]. Yuan, Y., Zou, W., Zhao, Y., Wang, X., Hu, X., Komodakis, N.: A robust and efficient approach to license plate detection. In: IEEE Transactions on Image Processing. Volume 26. (2017)

[10]. https://www.sighthound.com/

[11]. https://www.pyimagesearch.com/

\section{BIOGRAPHY}

1. Joel M John is a engineering student from Department of Computer Science and Engineering, at St Thomas College of Engineering and Technology Chengannur, Kerala under APJ Abdul Kalam Technological University. He is a passionate individual with experience in web development and python software programming with computer vision. He posses experience in hardware \& software integration and UI designing.

2. Noel Phillip Issac is a engineering student from Department of Computer Science and Engineering, at St Thomas College of Engineering and Technology Chengannur, Kerala under APJ Abdul Kalam Technological University. He posses good coding skills especially in python programming languages and other languages. He has practical experience in computer vision and good interpersonal skills.

3. Jerin Thomas is a engineering student from Department of Computer Science and Engineering, at St Thomas College of Engineering and Technology Chengannur, Kerala under APJ Abdul Kalam Technological University. $\mathrm{He}$ is a strong individual with analytical skills and problem solving capabilities. He posses interest in system testing and debugging tools in software development.

4. Subin Alexander is a engineering student from Department of Computer Science and Engineering, at St Thomas College of Engineering and Technology Chengannur, Kerala under APJ Abdul Kalam Technological University. $\mathrm{He}$ is an ambitious person and a good team player. He has developed outstanding approaches in problem solving especially in software development.

5. Syamraj B S is an Assistant Professor from Department of Computer Science and Engineering, at St Thomas College of Engineering and Technology Chengannur, Kerala under APJ Abdul Kalam Technological University. He posses his masters degree specialized in computer science and has strong analytical skills in Micro Processors and software programming. He has more than 4 years of experience and has published four papers in his teaching career. He has interest in Computer Networks, Microcontrollers, Microprocessors and Network programming. 